Diánoia, vol. 29, no. 29, 1983

\title{
EL MÉTODO DE ARQUÍMEDES
}

\author{
Adolfo García de la Sienra * \\ Universidad Michoacana de San Nicolás de Hidalgo, \\ MORELIA, MEXICO.
}

A Ricardo Arechavala

Los tratados de geometría griegos están escritos en conformidad con cánones metodológicos muy estrictos. Al igual que los Elementos de Euclides, casi todos ellos consisten en una cadena de proposiciones cuya demostración se omite únicamente cuando son tomadas como axiomas. Esta forma de presentar los resultados geométricos está bastante de acuerdo con el ideal griego de una episteme, pero ha tenido la desventaja - para los matemáticos e historiadores posteriores de la matemáticade ocultar completamente los procesos de construcción o descubrimiento de los conceptos y teorías elaborados por los geómetras griegos: como señala Knorr, ${ }^{1}$ muchos de los geómetras del siglo xviI, entre ellos Torricelli y el mismo Descartes, se quejaron de la opacidad de los métodos formales antiguos y sospechaban que los antiguos poseían métodos heurísticos que ocultaban de una manera deliberada. En particular, durante muchos siglos, matemáticos e historiadores de la matemática sospecharon que Arquímedes había aplicado en su descubrimiento de teoremas geométricos una técnica heurística de análisis de la cual, no obstante, ninguna traza se encontraba en los textos arquimedianos conocidos hasta el año de 1906. En el año de 1906 el investigador alemán J. L. Heiberg descubrió, en un antiguo palimpsesto conservado en la biblioteca del monasterio del Santo Sepulcro de Jerusalén examinado por él mismo en Constantinopla, un nuevo texto arquimediano que se consideraba perdido $^{2}$ en el que Arquímedes expuso un método para la "investigación" ( $\Theta \varepsilon \omega \varrho \varepsilon i v)$ de teoremas geométricos. El texto es designado allí como

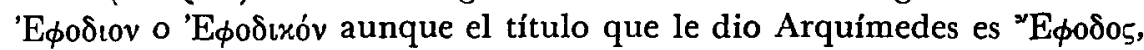
término que puede ser traducido como 'El método'. El método (EM) se encontraba junto a fragmentos de otros tratados arquimedianos ya

* Agradezco al profesor Wilbur R. Knorr, del Departamento de Clásicos de la Universidad de Stanford, los valiosos comentarios y sugerencias que me hizo cn conexión con este trabajo.

1 Ver Knorr (1978) en la Bibliografía.

= Sólo se sabía de su existencia por referencias de escritores antiguos. Suidas lo menciona y Herón lo cita en su Métrica (Heronis Opera III, 80, 84, 130). 
conocidos y, al igual que éstos, parcialmente borrado y cubierto por un texto más reciente -escrito entre los siglos xII y xIv- que contenía un eucologio. Afortunadamente, el texto original estaba casi completo y además era legible, lo que le permitió a Heiberg transcribirlo y dar a conocer al mundo un tesoro matemático que había permanecido oculto durante muchos siglos. ${ }^{3}$ Invito al lector a que me acompañe a continuación a admirar este tesoro. En primer lugar trataremos de ver en general en qué consiste y qué tipos de problemas permitía abordar, en seguida haremos un análisis de sus fundamentos y, por último, lo veremos en operación mediante un análisis detallado de la Proposición I de El método.

\section{Las caracteristicas generales del método}

El método no es un tratado ordinario de geometría griega. A diferencia de tales tratados, que jamás proponen un teorema sin acompañarlo de una correspondiente demostración basada exclusivamente en resultados previamente establecidos y aceptados por la comunidad de matemáticos griegos, EM expone cierto procedimiento mediante el cual el más grande matemático de la antigüedad clásica parece haberse convencido de la verdad de proposiciones importantes antes de buscar una demostración canónica de las mismas. EM contiene "investigaciones" de teoremas incluidos y debidamente demostrados en Cuadratura de la parábola, Sobre la esfera y el cilindro I y II, Sobre conoides y esferoides y en un tratado perdido sobre el centro de gravedad de los sólidos. ${ }^{4}$ No hay duda de que Cuadratura de la parábola es anterior a EM, pues en la Proposición 1 de este tratado Arquímedes alude a la demostración canónica de la misma, la cual se encuentra precisamente en el primer texto. Heath (1921) ubica a EM antes que Sobre la esfera y el cilindro I y II, y Sobre conoides y esferoides, pero la argumentación de Knorr (1978) al respecto deja poca duda de que EM es el último de los escritos estantes producidos por Arquímedes. Así, EM resultarla ser una presentación tardía de un método heurístico empleado por el mismo Arquímedes para des-

3 Heiberg reportó su descubrimiento y publicó el texto en su "Eine neue Archimedeshandschrift", Hermes XLII, 1907. T. L. Heath agregó una traducción inglesa de $E l$ método como suplemento a la segunda edición (1912) de The Works of Archimedes, publicada por primera vez en 1897. Este es el texto que utilizo aqui, pero también hay una edición castellana de EM, debida a José Babini, por la Editorial Universitaria de Buenos Aires.

4 Arquimedes se refiere a este texto como Los equilibrios ('Ioogeoríals) en Sobre los cuerpos flotantes II. Knorr conjetura que debib haber sido compuesto por Arquimedes entre Sobre conoides y esferoides y Sobre los cuerpos flotantes I. Cfr. Knorr (1978), p. 528 y Knorr (1978 a). 
cubrir o establecer proposiciones demostradas en tratados escrițos y publicados con anterioridad.

Los problemas que sabemos Arquímedes resolvió con su método son de tres tipos, a saber: 1) mostrar que el área o volumen de una figura geométrica no rectilínea se halla en cierta proporción con el área o volumen de una figura rectilínea, 2) mostrar que los volúmenes de dos figuras no rectilíneas se hallan en cierta proporción y 3) determinar el centro de gravedad de un sólido no rectilíneo. La Proposición 1 de EM es el único ejemplo del primer tipo; en ella se propone mostrar Arquímedes que el área del segmento de parábola $\mathrm{ABC}$, acotado por la línea recta AC y la parábola (también designada por) $\mathrm{ABC}$, es igual a $4 / 3$ el área del triángulo inscrito en el segmento (ver Figura 1).

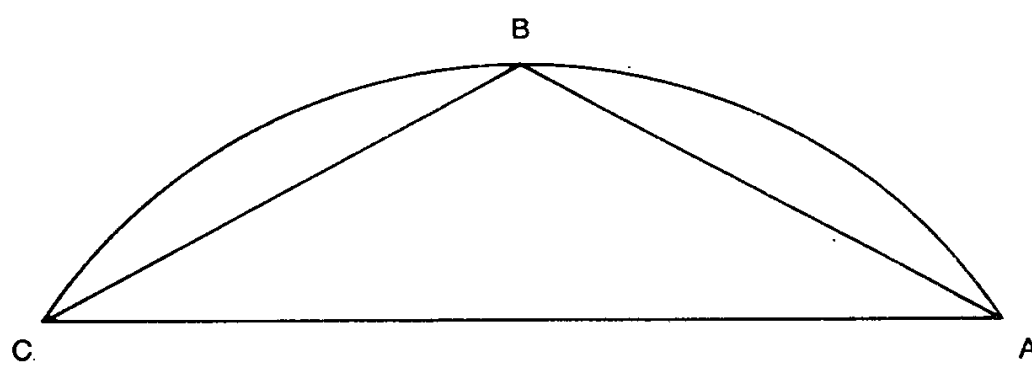

Figura 1.

Como un ejemplo del segundo tipo podemos mencionar la primera parte de la Proposición 2 de EM, donde se investiga la aserción de que "cualquier esfera es [con respecto al contenido sólido] cuatro veces el cono con base igual a un gran círculo de la esfera y altura igual a su radio". ${ }^{5}$ Un ejemplo del tercer tipo se encuentra en la Proposición 6, la que afirma que "el centro de gravedad de cualquier hemisferio [se halla en la línea recta que] es su eje, y divide la dicha línea recta de tal modo que la porción de ella adyacente a la superficie del hemisferio está con la restante porción en la misma razón que 5 está con $3 " .{ }^{6}$ Los tipos (1) y (2) se distinguen entre sí sólo por el hecho de que en el primer caso se establece una relación de proporcionalidad entre (el área de) una figura rectilinea y (la de) una curvilinea, mientras que en el segundo caso se muestra la proporcionalidad de (el volumen de) dos figuras curvilineas. Esta diferencia es importante, pues los geómetras griegos consideraban que el problema de determinar el área o volumen de una figura geométrica dada quedaba resuelto en el momento en que se demostraba la

5 Heath (1912), p. S-18.

6 Heath (1912), p. S-27. 
igualdad de (el área o volumen de) esa figura con (el área o volumen de) un cuadrado o un cubo. De ahí se deriva el famoso problema de la cuadratura del círculo, i. e., el problema de construir un cuadrado de área igual a la de un círculo dado. Sabemos que este problema es irresoluble mediante el método constructivo de la regla y el compás, pero Arquímedes demostró pọr reducción al absurdo que "el área de cualquier círculo es igual a un triángulo rectángulo en el cual uno de los lados adyacentes es igual al radio y el otro a la circunferencia del círcu10".7 Este resultado, junto con la estimación que hizo de la razón $\pi$ de la circunferencia de cualquier círculo a su diámetro, como una entre $3^{10} / 71(\approx 3.1408)$ y $3^{1} / 7(\approx 3.1428),{ }^{8}$ le permitió a Arquímedes calcular con un grado razonable de aproximación el área de cualquier círculo. La medición del círculo es un pilar fundamental de la obra de Arquímedes, porque ella le abrió la posibilidad de calcular las áreas y volúmenes de varias figuras curvilíneas importantes, como la esfera. Así, por ejemplo, en la Proposición 34 de Sobre la esfera y el cilindro I Arquímedes demuestra canónicamente la Proposición 2 de EM, arriba mencionada. Ahora bien, ya Euclides sabía que "cualquier cono es una tercera parte del cilindro que tiene su misma base e igual altura" (Euclides XII, 10), ${ }^{9}$ de donde se deduce que "todo cilindro cuya base es el círculo mayor de una esfera y cuya altura es igual al diámetro de la esfera es $3 / 2$ de la esfera" (Corolario a la Proposición 34 de Sobre la esfera y el cilindro I). - Por otra parte, la Proposición 14 del Libro II de los Elementos de Euclides permite construir un cuadrado igual a una figura rectilinea dada, en particular igual a un triángulo rectángulo. De esta manera, si $E$ es una esfera dada, $C$ es un círculo mayor y $d$ es el diámetro de la misma, los resultados en Medición de un círculo nos permiten "construir" un triángulo con lados adyacentes aproximadamente iguales respectivamente al radio de $C$ y a su circunferencia, y, por lo tanto, de área aproximadamente igual a la de $C$. A partir de aquí es posible obtener, mediante los otros resultados mencionados, un paralelepípedo sólido cuya base es un cuadrado aproximadamente igual a $C$ y cuya altura es igual al diámetro de la esfera $E$, i. e., un paralelepípedo cuyo volumen es aproximadamente igual al de un cilindro cuya base es el círculo mayor de la esfera $E$ y cuya altura es el diámetro $d$ de la misma. Está claro que el volumen de semejante paralelepípedo deberá ser aproximadamente igual a $3 / 2$ el volumen de la esfera, con lo que se obtiene una razón aproximada entre el volumen de la esfera y el de una figura rectilínea. De esta manera se

7 Proposición 1 de Medición de un circulo; cfr. Heath (1912), p. 91.

8 Proposición 3 de Medición de un círculo; Heath (1912), p. 93.

9 Sigo la edición en tres volúmenes de los Elementos debida a Heath (1956). El numeral romano se refiere al libro y el arábigo a la proposición. 
ve la pertinencia que tienen las comparaciones de áreas o volúmenes de figuras curvilineas en la obra de Arquímedes, pues ellas sirven como eslabones de una cadena de resultados que permiten, finalmente, establecer proporciones entre las áreas o volúmenes de figuras curvilíneas y rectilineas, i. e., que permiten cuadrar o "cubicular" las primeras.

$\mathrm{Al}$ lector le puede parecer que semejante modo de determinar el área o volumen de una figura es torpe y complicado, pero debe tener presente que la geometría griega no es algebraica: para los griegos las áreas, volúmenes y longitudes de las figuras geométricas no eran números, sino entidades de cierta naturaleza (¿espaciales?) cuyas propiedades y relaciones debian ser estudiadas con métodos adecuados a esa misma naturaleza. Gracias a la invención de la geometría analítica y sobre todo al desarrollo del cálculo diferencial e integral, las proposiciones que Arquímedes investigó con su método y demostró penosamente por procedimientos indirectos admiten en la actualidad una prueba muy directa e inmediata. Con el objeto de hacer comparaciones veremos enseguida la demostración contemporánea de la primera parte de la Proposición 2 de EM, así como la de la Proposición 6; ambas proposiciones fueron enunciadas más arriba.

En el primer caso se trata de mostrar que cualquier esfera es cuatro veces el cono con base igual a un gran círculo de la esfera y altura igual a su radio $r$. Para tal efecto, considérese a la esfera como el producto de una revolución, alrededor del eje $x$, del semicírculo acotado por el eje $x$ y la curva $y=+\sqrt{r^{2}-x^{2}}$. (Figura 2.)

Considérese asimismo al cono en cuestión como producto de la revolu-

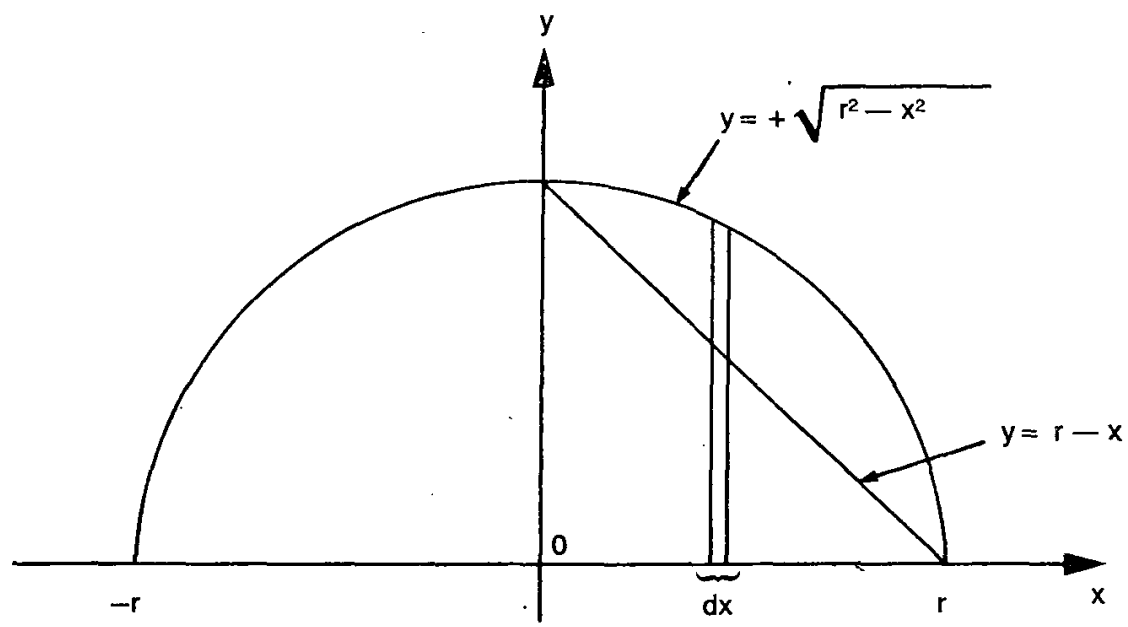

Figura 2. 
ción, también alrededor del eje $x$, del triángulo acotado por los ejes $x$ y $y$, asi como por la recta $y=r-x$. Dividanse ahora de manera exhaustiva tanto el semicírculo como el triángulo en bandas de anchura infinitesimal $d x$ y alturas $y=\sqrt{r^{2}-x^{2}}$ y $y=r-x$, respectivamente. Al efectuar estas bandas una revolución alrededor del eje de las abscisas generan discos cuyos volúmenes son $\pi\left(\sqrt{r^{2}-x^{2}}\right)^{2} d x$ en el primer caso, y $\pi(r-x)^{2} d x$ en el otro. Como los volúmenes de la esfera y del cono resultan de la "suma" (integral) de tales discos, obtenemos

$$
\begin{aligned}
\text { volumen de la esfera } & =\pi \int_{-r}^{r} r^{2}-x^{2} d x \\
& =\pi\left[r^{2} x-\frac{1}{3} x^{3}\right]^{r}-r \\
& =\frac{4}{3} \pi r^{3} \\
\text { volumen del cono } & =\pi \int_{0}^{r}(r-x)^{2} d x \\
& =\pi\left[r^{2} x-r x^{2}+\frac{1}{8} x^{3}\right]^{r} 0 \\
& =\frac{1}{3} \pi r^{3}
\end{aligned}
$$

Por lo tanto, el volumen de la esfera es cuatro veces el volumen del cono, como se había afirmado.

La demostración de la otra proposición involucra la definición del concepto de centro de gravedad de un sólido. Conviene considerar a nuestro hemisferio como el sólido generado por la revolución, alrededor del eje $y$, del cuarto de ćrculo acotado por los ejes $x$ y $y$, así como por la curva $x=\sqrt{r^{2}-y^{2}}$ (Figura 3). De esta manera, por razones de sime-

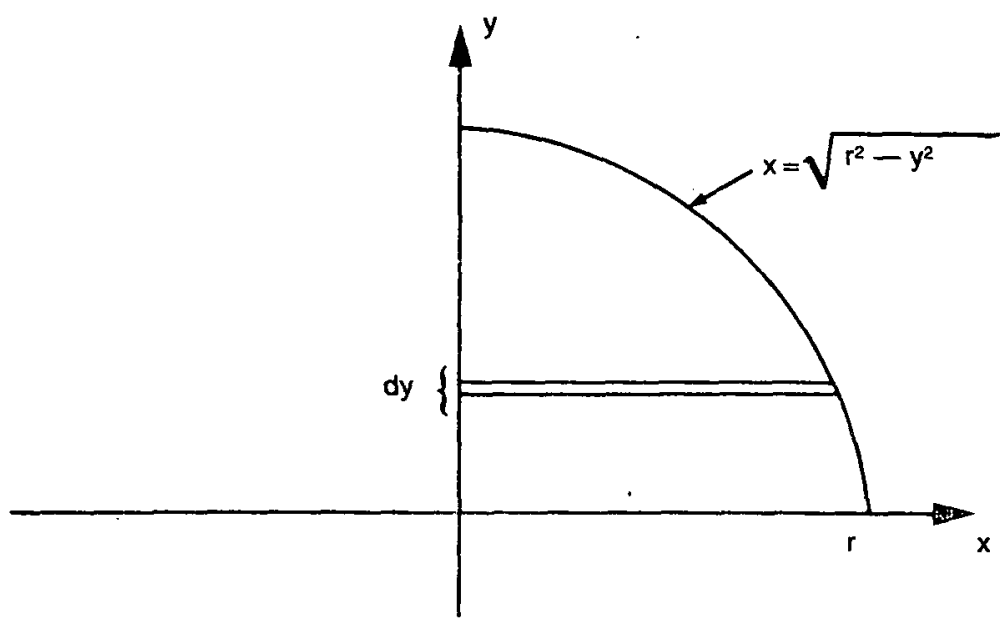

Figura 3. 
tría, el centro de gravedad está en el eje $y$ y, por definición, la coordenada $y$ del centro de gravedad es igual a

$$
\bar{y}=\frac{1}{V} \int_{0}^{r} \pi y\left(\sqrt{r^{2}-y^{2}}\right)^{2} d y
$$

donde $\mathrm{V}=\frac{2}{8} \pi r^{3}$ es el volumen del hemisferio. Así,

$$
\begin{aligned}
\bar{y} & =\frac{3 \pi}{2 \pi r^{3}} \int_{0}^{r} r^{2} y-y^{3} d y \\
& =\frac{3}{2 r^{3}}\left[\frac{1}{2} r^{2} y^{2}-\frac{1}{4} y^{4}\right]_{0} \\
& =\frac{3 r^{4}}{8 r^{3}} \\
& =\frac{3}{8} r
\end{aligned}
$$

Esta es la distancia del origen al centro de gravedad; la porción del eje hemisférico adyacente a la superficie del hemisferio es por consiguiente igual a $r-\frac{8}{8} r=\frac{\pi}{8} r$, de donde se deduce que la porción adyacente al hemisferio es a la porción restante lo que 5 es a 3, como había dicho el matemático de Siracusa.

El concepto de centro de gravedad no es geométrico sino más bien mecánico. Arquímedes escribió algunos tratados mecánicos entre los que cabe destacar Sobre el equilibrio de los planos I y II. El método de Arquímedes está basado principalmente en ese tratado y es por ello que se trata de un método mecánico; de un método mecánico, empero, utilizado principalmente para resolver problemas geométricos. La concepción del método es en extremo ingeniosa y original. En términos generales, el método consiste en lo siguiente. Dadas dos figuras A y B, Arquímedes las colocaba "empalmadas" en una cierta posición, de manera que establecía una correspondencia biunívoca entre sus "componentes" (segmentos rectilíneos, si eran figuras planas, o figuras planas, si eran sólidos). A continuación prolongaba Arquímedes uno de los ejes comunes a las figuras hasta un cierto punto $H$ e, imaginándose ese eje prolongado como los brazos de una palanca, designaba un punto $F$ sobre el mismo como fulcro. En seguida pasaba Arquímedes a mostrar que el "peso" (= longitud o área) de cada componente de $A$, ubicado a una distancia $D$ del fulcro $F$ en el lado opuesto a $H$ (es decir, donde se encuentra originalmente), es al "peso" (= longitud o área) del correspondiente componente de $B$ lo que la distancia $F H$ es a la distancia $D F$. En este momento aplicaba 
Arquimedes un resultado contenido en las proposiciones 6 y 7 de Sobre él equilibrio de los planos I, según el cual dos magnitudes en una palanca se equilibran a distancias recíprocamente proporcionales a sus magnitudes, para concluir que el componente de $A$, ubicado en $D$ que es donde se encuentra originalmente, balancea al correspondiente componente de $B$ puesto con su centro de gravedad en $H$. Esto lo establecía Arquímedes, de manera general, para todos y cada uno de los componentes de $A$ y $B$, con el objeto de deducir que $A$, donde originalmente se encontraba, balanceaba a $B$ colocado con su centro de gravedad en el extremo $H$ de la balanza. Una vez alcanzado este estadio en la investigación del problema, podía Arquímedes establecer uno de dos tipos de resultados finales: si el centro de gravedad de $A$ era conocido, Arquímedes podia establecer una comparación entre el área o el volumen de las figuras, del tipo " $A$ es igual a tantas veces $B$ " o, si era ésta la relación conocida, Arquímedes podía determinar el centro de gravedad de $A$. En algunas ocasiones, sin embargo, lo que le interesaba a Arquímedes no era tanto establecer la relación entre $A$ y $B$, cuanto establecer una proporcionalidad entre una parte $A^{\prime}$ de $A$ y $B$; en estos casos lo que hacia Arquímedes era aplicar teoremas geométricos ya establecidos para mostrar la existencia de una cierta proporción entre $A^{\prime}$ y $A$, y así, por transitividad, entre $A^{\prime}$ y. $B$. En esto consiste esencialmente el método de Arquímedes. Como se ve, presenta una semejanza con los métodos modernos de integración, precisamente en cuanto a la idea de concebir las figuras como compuestas de figuras ("bandas" o "discos") de anchura despreciable. Sin embargo, como veremos en la sección siguiente, se trata de una concepción diferente de la que prevalece en el análisis matemático contemporáneog.

\section{Los supuestos del método}

Es obvio que las investigaciones de las proposiciones llevadas a cabo por Arquímedes en EM requieren un uso sistemático de la teoría de las proposiciones, así como de otros resultados geométricos bien conocidos (y establecidos) en la época de Arquímedes. Casi todos estos resultados habían sido ya sistematizados por Euclides en sus Elementos, pero Arquímedes tomó también algunas proposiciones de los Elementos de las cónicas (un trabajo previo debido a Aristeo o Euclides) cuando escribió EM, pues en la investigación de la Proposición 1, cuando aplica un cierto resultado geométrico como lema, ${ }^{10}$ Arquímedes dice que lo tomó precisamente de ese tratado. Al comienzo de EM Arquímedes enunció nueve lemas, afirmando que "todas estas proposiciones habían sido ya demostradas". Sin embargo, solamente seis de ellas aparecen en alguna

10 Ver el lema EMI en el Apéndice. 
de sus obras estantes, presumiendose que las tres restantes fueron demostradas en tratados ahora perdidos. ${ }^{11}$ Como quiera que sea, Arquímedes consideraba a esas proposiciones como hechos geométricos bien establecidos. Ahora bien, lo que es peculiar de EM es que en este tratado Arquímedes adoptó también ciertas suposiciones, de gran valor heurísiico, pero que nunca fueroñ aceptadas por los geómctras gricgos - ni siquiera por el mismo Arquímedes_ como verdades establecidas. Estas suposiciones son tres:

(S1) Una figura plana está compuesta por segmentos de rectas y un volumen por figuras planas.

(S2) Sea $A_{i}(i \in I)$ una serie de magnitudes, $B_{i}(i \in I)$ una partición de la magnitud $B=\Sigma_{i \in I} B_{i}, D_{i}(i \in I)$ una colección de puntos sobre el brazo de una palanca con fulcro $F$ y $H$ un punto en el otro brazo. Si, para cada $i \in I$, la magnitud $A_{i}$ es a $B_{i}$ lo que la distancia $F H$ es a $D_{i} F$, entonces las magnitudes $A_{i}$, colocadas con su centro de gravedad de manera correspondiente en los puntos $D_{i}$, balancean alrededor de $F$ a $B$ colocada con su centro de gravedad en $H$.

(S3) Si un número cualquiera de magnitudes $A_{i}(i \in I)$, colocadas con sus centros de gravedad en los puntos $D_{i}$ de una palanca, equilibran alrededor del fulcro $F$ una magnitud $B$ colocada con su centro de gravedad en un punto $H$ situado en el extremo del otro brazo de la palanca, entonces la magnitud $\Sigma_{i \in r} A_{i}$, colocada en el centro de gravedad del sistema original $A_{i}$, balancea a $B$ colocada donde está.

Éxaminaremos separadamente cada una de estas suposiciones.

Con respecto a S1. Arquimedes claramente supone que una figura geométrica está hecha a partir de un número infinito de figuras de la dimensión inferior inmediata, de manera que la suma (infinita) de las magnitudes de estas figuras es igual a la magnitud de la figura completa. Por ejemplo, Arquímedes diría que el triángulo $A B C$ puede ser descompuesto en un número infinito de segmentos $D E$, paralelos a $A B$, cada uno de los cuales tiene como "base" un punto sobre $B C$. Análogamente, Arquímedes admitiría que el cilindro $F$. está hecho a partir de un número infinito de círculos $K$, paralelos e iguales a la base del cilindro (Fig. 4). Así, para Arquímedes un segmento de línea recta aparece como una banda de anchura muy pequeña y un circulo como un disco de espesor despreciable aunque no nulo.

11 Posiblemente en Los equilibrios. 


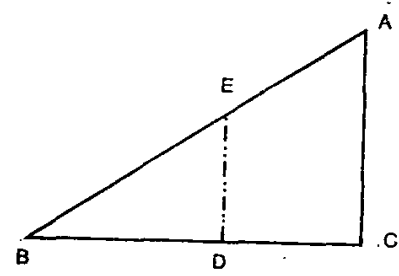

Figura 4.

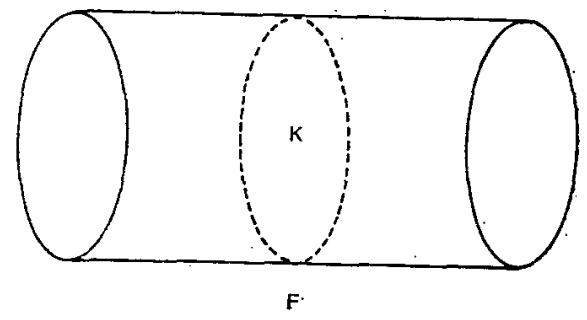

Semejante concepción de las figuras geométricas no era muy ortodoxa dentro de la tradición matemática griega. En los Elementos, Euclides "define" un punto como aquello que carece de partes. Heath dice que esta caracterización del punto puede ser entendida en el sentido de que un punto es aquello que es indivisible en partes. ¿Se sigue de ello que la extensión de un punto es nula? Martianus Capella (s. v a. C.) tradujo la "definición" euclideana de punto como "Punctum est cuius pars nihil est" pero, como Heath subraya, "si una parte de un punto no es nada, Euclides bien podía haber dicho que un punto no es él mismo 'nada', lo que desde luego no hace". ${ }^{12}$ Supongamos, entonces, que un punto es "algo". Ahora bien, hay un sentido obvio en el que estas entidades llamadas "puntos" carecen de extensión, a saber, si "extensión" es tomado en el sentido euclideano usual de longitud, anchura o profundidad. Pues si un punto tuviera (digamos) longitud, entonces sería una línea y, por lo tanto (infinitamente) divisible, lo que contradice la misma "definición" de punto. Por otro lado, la suposición de que la extensión de un punto es nula implica que cualquier suma de puntos, inclusive una infinita, debe ser igual a cero. Pero si éste es el caso, entonces la longitud de una línea recta no puede ser concebida como la suma de las extensiones de sus puntos. Esta es la razón por la que Aristóteles dijo que una línea no está hecha a partir de puntos -0 indivisibles-, pues de acuerdo con él la extensión de un punto es cero, como se puede ver fácilmente en el siguiente pasaje:

[...] no hay razón de cero a un número. Pues si 4 excede a 3 por 1 , y a 2 por más de 1 , y a 1 por más aun de lo que excede a 2 , aun asi no hay una razón por la que excede a 0 ; pues aquello que excede debe ser divisible entre el exceso + aquello que es excedido, de modo que 4 será lo que excede a 0 por +0 . Por esta razón, también, una línea no excede a un punto - ia menos que esté compuesta de puntos! ${ }^{13}$

12 Comentario a la Definición 1 de los Elementos; v. I, pp. 155-158.

13 Física, 215 b 12. 
Claramente, la suposición de que la anchura de una línea y la profundidad de un plano son también nulas, conduce a resultados análogos, i. e., implica que ni un plano está constituido por líneas, ni un volumen por figuras planas.

Curiosamente, a pesar de los esfuerzos de Aristóteles por mostrar su incorrección, la concepción arquimediana de las figuras geométricas conduce a cómputos precisos. Por ejemplo, sea $A B C$ un triángulo tal que $B C$ es a $A B$ lo que 1 es a 2 (Fig. 5) y suponga que cada uno de los puntos a partir de los cuales $A B$ está hecha, tiene una longitud muy pequeña $\varepsilon$. Tome $A B$ como unidad y sea $N=1: \varepsilon$ la razón de $A B$ a cualquiera de sus puntos. Se sigue que $\varepsilon=1 / N$ y que la altura de la linea recta $X Y$, perpendicular a $A B$ y que tiene a $X$ como "base", satisface la proporción

$$
\begin{aligned}
X Y: A X & =B C: A B \\
& =1: 2 .
\end{aligned}
$$

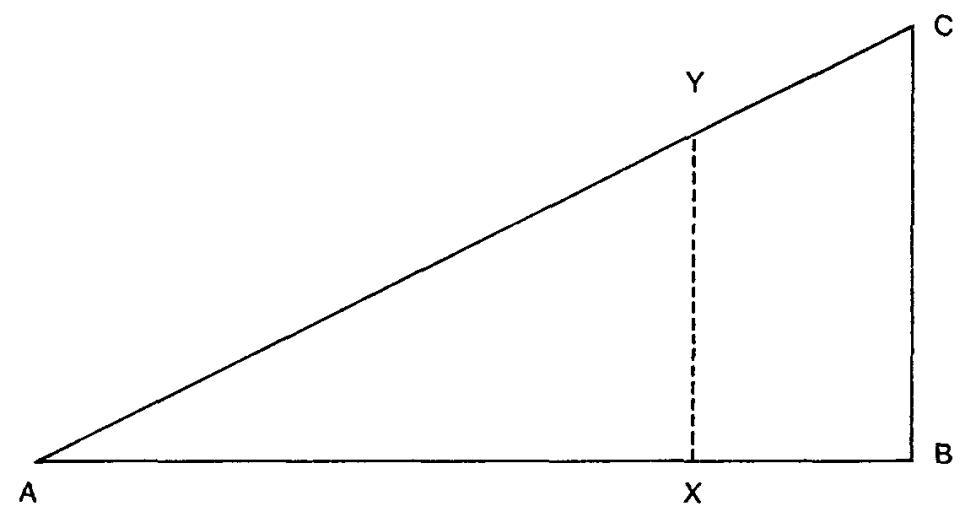

Figura 5.

Luego,

$$
X Y=A X / 2
$$

y el "área" del pequeño "rectángulo" sobre $X$ es

$$
A X / 2 \cdot 1 / N
$$

Así, de acuerdo con la idea de Arquímedes, el área de $\triangle A B C$ debe ser igual a la suma

$$
\Sigma_{\mathrm{X}}\left(\frac{1}{2} \cdot A X \cdot 1 / N\right) \quad(X \text { es un punto sobre } A B) .
$$


Dése cuenta, sin embargo, de que longitud de $A X=$ número de puntos en $A X \cdot 1 / N$, i.e.,

$$
A X=v / N,
$$

donde $v$ es el número de puntos sobre $A X$. Así, el área de $\triangle A B C$ es igual a

$$
\begin{aligned}
\sum_{v=1}^{N}\left(\frac{1}{2} \cdot v / N \cdot 1 / N\right) & =\sum_{v=1}^{N}\left(\frac{1}{2} \cdot v / N^{2}\right) \\
& =\frac{1}{2} N^{2} \cdot \sum_{v=1}^{N} v \\
& =\frac{1}{2} N^{2} \cdot N(N+1) / 2^{14} \\
& =\frac{1}{4} \cdot N(N+1) / N^{2} \\
& =\frac{1}{4} \cdot(N+1) / N \\
& =\frac{1}{4} \cdot(N / N+1 / N) \\
& =\frac{1}{4} \cdot(1+1 / N) \\
& =\frac{1}{4}+\left(\frac{1}{4} \cdot 1 / N\right) \\
& =\frac{1}{4}+\varepsilon / 4
\end{aligned}
$$

Por otra parte, un cálculo trivial muestra que el área de $\triangle A B C$ es $\frac{1}{4}$. Así, jel resultado alcanzado mediante el procedimiento de la suma infinita se desvía del valor exacto del área del triángulo sólo por "un cuarto" de punto! Este es desde luego un resultado muy notable. Pero no hay nada en el cómputo anterior que le hubiera impedido a Arquímedes realizarlo. Es posible que Arquímedes haya puesto a prueba su suposición $S 1$ haciendo algunos cómputos de este tipo, obteniendo el área o volumen (previamente conocido) de algunas figuras de fácil manipulación. Como quiera que haya sido, el método de la suma directa, aun si fuera aplicable a figuras más complicadas, no era suficiente para resolver el problema de determinar el área o volumen de una figura dada. Pues tal problema requiere de una solución general, i. e., encontrar no el valor numérico de esta área o volumen en particular, sino una relación general y fija entre todas las figuras de cierto tipo (e. g., círculos) y otra figura de un tipo fijo (e. g., el cuadrado). Como dijimos, encontrar esta solución significaba "cuadrar la figura". En la actualidad determinamos el área o volumen de una clase dada de figuras encontrando la integral indefinida de cierta función. Por ejemplo, podemos de-

14 Cfr. el lema a la Proposición I de Sobre conoides y esferoides. 
terminar el área de todos los triángulos similares a $\triangle A B C$, integrando la función $y=\frac{1}{2} x$ :

$$
\int \frac{1}{2} x d x=\frac{1}{4} x^{2}+C .
$$

El teorema fundamental del cálculo nos permite entonces encontrar el valor numérico particular del área de este o aquuel criángüulo. Por ejemplo, el área de $\triangle A B C$ se calcula simplemente haciendo las sustituciones adecuadas:

$$
\left[\frac{1}{4} x^{2}\right]_{0}^{1}=\frac{1}{4}
$$

Pero el desarrollo de este procedimiento requirió de la invención de la geometria analítica y de unos cuantos siglos de penosos esfuerzos. Lo más que podían hacer los griegos para determinar en general el área o volumen de una figura geométrica ( $\mathrm{y}$ así es como el problema era propuesto); era describir un procedimiento general mediante el cual no podía cons: truir un área o volumen rectilíneos (como un cuadrado o un cubo) igual al primero. Esto es algo que ya habíamos discutido en el $\S 1$.

Debe ser subrayado que - a pesar de la corrección del resultado que obtuvimos mediante el procedimiento de la suma infinita- la descripción del proceso de cálculo es conceptualmente errónea y puede conducir a extrañas paradojas. Bonaventura Cavalieri (1598-1647), por ejemplo, señaló el siguiente curioso resultado. Considere dos triángulos rectángulos con un lado común como se muestra en la Figura 6. Desde un punto arbitrario $X_{1}$ sobre $A B$ dibuje una paralela a $A C$ que intersecte a $B C$ en $X_{2}$. Desde $X_{1}$ dibuje una paralela a $B D$ que intersecte $A D$ en $Y_{1}$, y desde $X_{2}$ produzca $X_{2} Y_{2}$ paralela a $B D$. Esto establece una correspondencia biunívoca entre el conjunto de las líneas rectas que componen a $\triangle A B D$ y el de los segmentos de recta que componen a $\triangle B D C$. Dado que cada línea $x_{1} y_{1}$ es igual a su línea asociada $x_{2} y_{2}$, se sigue que $\triangle A B D=\triangle B D C$.

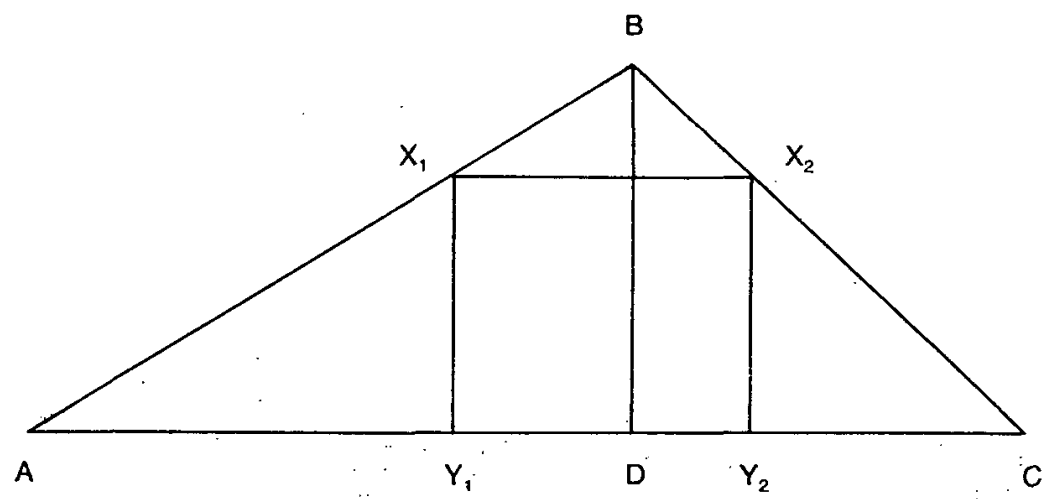

Figura 6. 
Pero esto es claramente falso. De hecho, sabemos que dos intervalos cualesquiera de números reales son equipolentes, de modo que en rigor es simplemente falso que una figura plana esté compuesta por segmentos de rectas o que un volumen lo esté por figuras planas. No obstante, el procedimiento de la suma infinita puede ser justificado teoréticamente, mediante ciertos resultados de la lógica matemática. Una discusión general de tales resultados nos llevaría muy lejos, pero podemos proporcionar una breve justificación del cómputo del área del triángulo, que llevamos a efecto arriba, utilizando estos resultados. ${ }^{15}$

Considere de nuevo el triángulo $A B C$, pero ahora como la gráfica de la función $f(x)=\frac{1}{2} x$. Tome a $A B$ como el intervalo $[0,1]$ sobre la línea real, con $A$ ubicado en el origen, y divida a $A B$ en $n$ intervalos iguales de longitud $\Delta x$. Sobre cada intervalo $\left[x_{i-1}, x_{i}\right]$ dibuje un rectángulo de altura $f\left(x_{i}\right)$ y observe que el área del rectángulo es $f\left(x_{i}\right) \cdot \Delta x$. Permita que $\int_{0}^{1} f(x) \cdot \Delta x$ denote la suma de las áreas de esos rectángulos y dése cuenta de que $S f(x) \cdot \Delta x$ es una función de una variable real $(\Delta x)$. Aho ra bien, para cualquier entero positivo real $n$, si $\Delta x=1 / n$, tenemos

$$
\begin{aligned}
\stackrel{S}{0}_{0}^{1} f(x) \cdot \Delta x & =\sum_{i=1}^{n} f\left(x_{i}\right) \cdot 1 / n \\
& =\sum_{i=1}^{n} f(i / n) \cdot 1 / n \\
& =\sum_{i=1}^{n}\left(\frac{1}{2} \cdot i / n \cdot 1 / n\right) \\
& =\sum_{i=1}^{n} i / 2 n^{2} \\
& =\frac{1}{2} n^{2} \cdot \sum_{i=1}^{n} i \\
& =\frac{1}{2} n^{2} \cdot n(n+1) / 2 .
\end{aligned}
$$

De esta manera hemos mostrado que el enunciado de primer orden

$$
\begin{aligned}
&{ }^{*} \forall x \forall y\left(\left(y \text { es un entero positivo \& } \frac{1}{y}=\Delta x\right)\right. \\
&\left.\rightarrow \operatorname{S}_{0}^{1} f(x) \cdot \Delta x=\frac{1}{2} y^{2} \cdot y(y+1) / 2\right),
\end{aligned}
$$

es verdadero en el campo de los números reales. Dado que este campo

15 Para una exposición introductoria al análisis no estándar, el lector puede acudir al texto de Henle y Kleinberg (1979), que es de donde he tomado la notación que uso enseguida. 
es elementalmente equivalente al campo (¡no arquimediano!) de los números hiperreales, se sigue que el enunciado es también verdadero en este último campo. Escoja un entero positivo infinito $N$, haga $1 / N=\Delta x$ $y$, especificando universalmente el enunciado de primer orden, reemplace a $y$ con $N$. Entonces obtenemos

$$
\begin{aligned}
\int_{0}^{1} f(x) \cdot \Delta x & =\frac{1}{2} N^{2} \cdot N(N+1) / 2 \\
& =\frac{1}{4}+\left(\frac{1}{4} \cdot 1 / N\right) \\
& =\frac{1}{4}+\varepsilon / 4
\end{aligned}
$$

donde $\varepsilon$ es el infinitesimal que es el inverso de $N$. Ahora bien, la suma infinita (la integral) de los rectángulos infinitamente delgados $f(x) \cdot \Delta x$ es por definición igual a la parte estándar de $\int_{0}^{1} f(x) \cdot \Delta x$, i. e., al número real más cercano a $S_{0} f(x) \cdot \Delta x$. Pero

$$
\operatorname{st}\left({ }_{0}^{1} f(x) \cdot \Delta x\right)=\frac{1}{4}
$$

porque $\varepsilon / 4$ es desde luego infinitesimal. Ésta es una descripción impecable y perfectamente rigurosa del procedimiento de la suma infinita, la cual no supone que una figura está hecha a partir de líneas o planos. De esta manera, si, como dice Heath (1912),

aunque Arquímedes llama a los elementos [de una figura geométrica] líneas rectas y áreas planas respectivamente, ellos son por supuesto, en el primer caso, bandas indefinidamente estrechas (áreas) $y$, en el segundo caso, láminas planas indefinidamente delgadas (sólidos), ${ }^{16}$

entonces podemos concluir que el procedimiento de la suma infinita, sugerido por la concepción arquimediana de las figuras geométricas, es esencialmente correcto, aunque la concepción misma sea errónea.

Con respecto a $S 2$. Una demostración canónica del caso infinito de $S 2$ era algo que se encontraba más allá del alcance de las técnicas matemáticas de los griegos. Más aún, no se necesita ser demasiado perspicaz para darse cuenta de que ni siquiera el caso más simple, el que involucra sólo cuatro magnitudes, puede ser demostrado a partir de la base primitiva propuesta por Arquímedes en sus tratados estantes de mecánica. Las definiciones y axiomas necesarios debían haber aparecido en Sobre el equi- 
librio de los planos $\mathrm{I}$, pero, como se sabe, el sistema axiomático allí introducido por Arquímedes es incompleto y defectuoso en algunos respectos. No obstante, Arquímedes utiliza el caso finito general para $n$ magnitudes de $S 2$ en las demostraciones de las proposiciones 14 y 15 de Cuadratura de la parábola, por lo que es obvio que Arquímedes estimaba que ese caso de $S 2$ era una verdad perteneciente a su teorfa de la palanca. En su reconstrucción de la teoría arquimediana del centro de gravedad, Stein (1935) introdujo el caso simple de $S 2$ como una "suposición tácita" de Arquímedes. Como quiera que sea, lo que Arquímedes utiliza en EM es una versión infinita que, como dije, no era posible demostrar con los recursos de que Arquímedes disponía.

La versión contemporánea de $S 2$ admite una demostración simple y directa. Veamos primero el caso finito. Para ello, imagínese usted que el eje de las $x$ en el plano cartesiano es una palanca con fulcro en el origen, considere $n$ magnitudes $A_{1}, \ldots, A_{n}$ y $n$ puntos $x_{1}, \ldots, x_{n}$ sobre el mismo lado del eje $x$. Si $h$ es un punto al otro lado del origen, $B_{1}, \ldots, B_{n}$ es una descomposición de una figura $B=\sum_{i=1}^{n} B_{i}$ y $A_{i}$ es a $B_{i}$ lo que $h$ es a $x_{i}$, entonces $x_{i} A_{i}=h B_{i}$ y

$$
\begin{aligned}
\sum_{i=1}^{n} x_{i} A_{i} & =h \sum_{i=1}^{n} B_{i} \\
& =h \mathrm{~B} ;
\end{aligned}
$$

i. e., las magnitudes $A_{i}$ colocadas correspondientemente en los puntos $\boldsymbol{x}_{\boldsymbol{i}}$ balancean alrededor del origen a $B$ colocada con su centro de gravedad en $h$. Para demostrar la versión infinita que necesitaba Arquímedes, considere dos figuras simétricas A y $\mathbf{B}$-planos o sólidos de revolucióncon sus ejes de simetría coincidiendo con el intervalo $I=[a, b]$ en el eje $x$, y descomponga estas figuras en segmentos — bandas o láminas-$\alpha(x) d x$ y $\beta(x) d x$ de espesor infinitesimal $d x$, utilizando las funciones continuas $\alpha$ y $\beta$ adecuadas. Si $-h$ es un punto sobre el eje $x$ al otro lado del origen, tal que $\alpha(x) d x$ es a $\beta(x) d x$ lo que $h$ es a $x$ para cada $x$ en $I$, entonces $x \alpha(x) d x=b \beta(x) d x$ y

$$
\begin{aligned}
\int_{a}^{b} x \alpha(x) d x & =h \int_{a}^{b} \beta(x) d x \\
& =h \mathrm{~B}
\end{aligned}
$$

de manera que la figura $\mathrm{A}$, donde está, balancea a $\mathrm{B}$ ubicado con su centro de gravedad en $-h$, alrededor del origen tomado como fulcro de una palanca. 
Con respecto a $S 3$. Al igual que $S 2, S 3$ es una suposición extraordinaria cuya demostración en el caso infinito era inaccesible para las técnicas de que podía disponer Arquímedes. Antes de pasar a la demostración contemporánea de los dos casos de $S 3$ —el finito y el infinitoofreceré al lector una prueba de $S 3$ para el caso más simple utilizando exclusivamente resultados y técnicas que estaban al alcance de Arrquímedes. Espero con esto sensibilizar al lector hacia las limitaciones técnicas de la época, las que hacían imposible una demostración canónica del caso general, así como transmitirle una idea del estilo de dercostración de Arquímedes en sus tratados mecánicos. Para demostrar ese caso de $S 3$ haré uso de axiomas y teoremas contenidos en Sobre el equilibrio de los planos I (EP I), asi como de tres "suposiciones tácitas" de Arquímedes, a saber:

(T1) Si $A$ y $B$ son magnitudes ubicadas en brazos diferentes de una palanca, entonces se da precisamente una de las siguientes situaciones: i) $A$ y $B$ están en equilibrio; ii) la palanca está inclinada hacia $A$; iii) la palanca está inclinada hacia $B$.

(T2) El caso finito de $S 2$.

(T3) Si $A$ y $B$ son magnitudes ubicadas en brazos diferentes de una palanca y $A$ es demasiado pesada para estar en equilibrio $\operatorname{con} B$, es posible quitar de $A$ una porción tal que la restante estará en equilibrio con $B$.

Stein (1935) califica también a T3 como una "suposición tácita" de Arquímedes y no menciona a Tl, aunque está claro que Tl también lo es, pues Arquímedes presupone esa tesis en la demostración de la importante Proposición 7 de EP I, la que afirma que dos magnitudes inconmensurables se balancean a distancias reciprocamente proporcionales a las magnitudes. Sin embargo, T1 no aparece ni como axioma ni como teorema en ninguno de los tratados estantes de Arquímedes; posiblemente se trate de una de esas suposiciones que los geómetras griegos adoptaban calladamente, precisamente debido a su obviedad. Utilizando tan obvias suposiciones demostraremos a continuación tres lemas previos al teorema que nos interesa.

Lema 1. Si dos magnitudes $A_{1}$ y $A_{2}$, ubicadas con sus centros de gravedad en $D_{1}$ y $D_{2}$, respectivamente, balancean alrededor del fulcro $F$ de una palanca a una tercera magnitud $B=B_{1}+B_{2}$, puesta con su centro de gravedad en un punto $H$ en el otro extremo de la palanca, y si la magnitud $A_{1}$ es a $B_{1}$ lo que la distancia $F H$ es a $D_{1} F$, entonces $A_{2}: B_{2}=$ $=F H: D_{2} F$.

Demostración. Retire $A_{1}$ de la palanca y reste $B_{1}$ a $B$. Entonces sólo hay tres posibilidades: ( $i$ ) $A_{2}$ equilibra a $B_{2}$, en cuyo caso no hay nada que 
demostrar; (ii) la palanca se inclina hacia $A_{2}$; (iii) la palanca se inclina hacia $B_{2}(T 1)$. Si la palanca se inclina hacia $A_{2}$, retire una porción $C$ de $A_{2}$ hasta que la palanca se nivele (T3), de modo que $A_{2}-C: B_{2}=F H$ : $D_{2} F$ (EP I, 6, 7). Entonces $S 2$ (= T2) implica que las magnitudes $A_{1}$ en $D_{1}, A_{2}-C$ en $D_{2}$ y $B$ en $H$, están en equilibrio alrededor de $F$, de donde se deduce que el sistema original $A_{1}$ en $D_{1}, A_{2}$ en $D_{2}, B$ en $H$, no lo está, pues la palanca debiera en tal caso estar inclinada hacia $A_{2}$ (EP I, Postulado 3), lo que contradice la hipótesis. De la misma manera se prueba que la palanca no puede estar inclinada hacia $B_{2}$. Por lo tanto, la palanca está en equilibrio, i. e., $A_{2}: B_{2}=F H: D_{2} F($ EP I, 6,7$)$.

Q. E. D.

Lema 2. Si dos magnitudes $A_{1}$ y $A_{2}$, ubicadas con sus centros de gravedad en $D_{1}$ y $D_{2}$, respectivamente, balancean alrededor del fulcro $F$ de una palanca a una tercera magnitud $B$, colocada con su centro de gravedad en un punto $H$ en el otro extremo de la palanca, entonces $B$ admite una descomposición en partes $B_{1}$ y $B_{2}$ tales que $B=B_{1}+B_{2}, A_{1}: B_{1}=$ $F H: D_{1} F$ y $A_{2}: B_{2}=F H: D_{2} F$.

Demostración. Retire $A_{2}$ de la palanca con lo que ésta se inclinará hacia $B$ (EP I, P.3), y a continuación retire una porción $B_{2}$ de $B$ hasta que la palanca se nivele (T3), de manera que $A_{1}: B_{1}=F H: D_{1} F\left(B_{1}=B-B_{2}\right)$ (EP I, 6, 7). Entonces $A_{2}: B_{2}=F H: D_{2} F($ Lema 1$)$ y $B=B_{1}+B_{2}$.

Q. E. D.

Lema 3. Sea $F$ el fulcro de una palanca, $D_{1}$ y $D_{2}$ puntos sobre uno de sus brazos, y $H$ un punto en el extremo del otro brazo. Si $A_{1}, A_{2}, B_{1}, B_{2}$ son magnitudes tales que
(1) $A_{1}: B_{1}=F H: D_{1} F$
$\mathrm{y}$
(2) $A_{2}: B_{2}=F H: D_{2} F$,

entonces la magnitud compuesta $A=A_{1}+A_{2}$, colocada en el centro de gravedad del sistema $A_{1}$ (en $D_{1}$ ), $A_{2}$ (en $D_{2}$ ), equilibra a la magnitud $B=B_{1}+B_{2}$ colocada con su centro de gravedad en $H$ (figura 7 ).

Demostración. Sea $E$ el centro de gravedad del sistema $A_{1}$ (en $D_{1}$ ), $A_{2}$ (en $D_{2}$ ), de modo que

(3) $A_{1}: A_{2}=D_{2} E: D_{1} E$. 


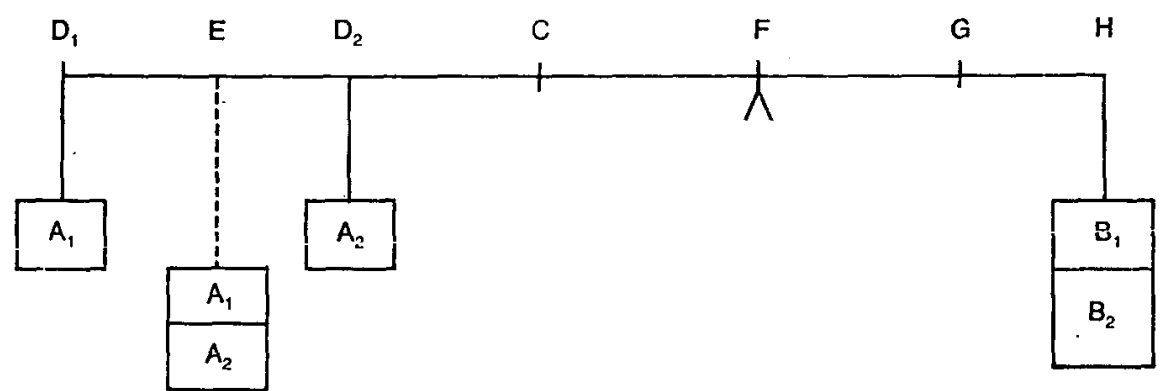

Figura 7.

Divida $\dot{F} H$ de acuerdo con la proporción $D_{2} E: D_{1} E$, encontrando un punto $G$ tal que

(4) $F G: G H=D_{2} E: D_{1} E$

y, de la misma manera, divida $E F$ encontrando $C$ tal que

(5) $F H: D_{1} F=F G: E C$

(Euclides VI, 10). Entonces $F H=F G+F M$ y

(6) $F G+G H: D_{1} F=F G: E C$

(7) $F G+G H: F G=D_{1} F: E C$

(alternando)

(8) $G H+F G: F G=\left(D_{1} E+C F\right)+E C: E C$

(9) $G H: F G=D_{1} E+C F: E C$

(separando)

(10) $F G: G H=E C: D_{1} E+C F$

(invirtiendo)

Luego, puesto que $E C=D_{2} E+D_{2} C$, (4) y (10) implican

(11) $D_{2} E+D_{2} C: D_{1} E+C F=D_{2} E: D_{1} E$

(12) $D_{2} E+D_{2} C: D_{2} E=D_{1} E+C F: D_{1} E$

(alternando)

(13) $D_{2} C: D_{2} E=C F: D_{1} E$

(separando)

(14) $D_{2} C: C F=D_{2} E: D_{1} E$

(alternando) 
(15) $\mathrm{D}_{2} \mathrm{C}: C F=F G: G H$

(16) $F G+G H: G H=D_{2} C+C F: C F$

(componiendo)

(17) $F H: \dot{G} H=D_{2} F: C F$

(18) $F H: D_{2} F=G H: C F$

(alternando)

(19) $G H: C F=A_{2}: B_{2}$

De esta manera, obtenemos las siguientes proporciones:

$$
\begin{aligned}
& A_{1}: B_{1}=F G: E C \\
& A_{2}: B_{2}=G H: C F \\
& A_{1}: A_{2}=D_{2} E: D_{1} E
\end{aligned}
$$

De aquí se deduce inmediatamente, mediante un lema demostrado por Arquímedes, ${ }^{17}$ que

$$
A_{1}+A_{2}: B_{1}+B_{2}=F G+G H: E C+C F
$$

o sea,

$$
A: B=F H: E F .
$$

Por lo tanto, la magnitud $A$ colocada en el centro de gravedad $E$ del sistema $A_{1}, A_{2}$ balancea a $B$ colocada en $H$ (EP I, 6, 7).

Q. E. D.

Una vez demostrados los lemas necesarios, podemos proceder a formular y a probar el caso elemental de $S 3$. Este es el siguiente

Teorema. Si dos magnitudes $A_{1}, A_{2}$, colocadas con sus centros de gravedad en los puntos $D_{1}, D_{2}$ (respectivamente) del brazo de una palanca, equilibran alrededor del fulcro $F$ una magnitud $B$ colocada con su centro de gravedad en un punto $H$ situado en el extremo del otro brazo de la palanca, entonces la magnitud $A_{1}+A_{2}$, colocada en el centro de gravedad $E$ del sistema original $A_{1}, A_{2}$, balancea a $B$ colocada donde está.

Demostración. Por el Lema 2, $B$ admite una descomposición en partes $B_{1}, B_{2}$ tales que $B=B_{1}+B_{2}, A_{1}: B_{1}=F H: D_{1} F$ y $A_{2}: B_{2}=F H: D_{2} F$. Luego, por el Lema $3, A_{1}+A_{2}$ ubicada en el centro de gravedad del sistema $A_{1}, A_{2}$ balancea a $B$ colocada en $H$.

Q. E. D.

17 Ver lema EM2 en el Apéndice. 
Demostrar el caso finito general de este teorema empleando exclusivamente técnicas antiguas es ùn ejercicio nada trivial que puede intentar el lector interesado en practicar sus conocimientos de geometría griega. En contraste, la demostración moderna de $S 3$ es simple y directa. Para probar el caso finito general, considere al eje de las $x$ como una palanca con fulcro en el origen y observe que $n$ magnitudes $A_{1}, \ldots, A_{n}$ balanccan a una magnitud $A=\sum_{i=1}^{n} A_{i}$ colocada en el punto $-\bar{x}$, alrededor de 0 , si

i. e., si

$$
-\bar{x} A+\sum_{i=1}^{n} x_{i} A_{i}=0
$$

$$
\bar{x}=\frac{1}{A} \sum_{i=1}^{n} x_{i} A_{i},
$$

de manera que las $n$ magnitudes originales $A_{1}, \ldots, A_{n}$ actúan sobre la palanca como si fueran una sola magnitud colocada en $\bar{x}$. Así definido, $\bar{x}$ es el centro de gravedad del sistema $A_{1}, \ldots, A_{n}$. Ahora bien, lo que hay que demostrar es que si estas magnitudes, ubicadas en $x_{1}, \ldots, x_{n}$ balancean alrededor del origen a una magnitud $B$ colocada en $-h$, entonces $A$ colocada en $\bar{x}$ balancea a $B$ colocada en $-h$ alrededor de 0 . La hipótesis de esta aserción es verdadera si, y sólo si, $\sum_{i=1}^{n} x_{i} A_{i}=h B$; pero

$$
\bar{x} A=\frac{1}{A} \sum_{i=1}^{n} x_{i} A_{i} \cdot A=\sum_{i=1}^{n} x_{i} A_{i}=h B,
$$

con lo que se cumple también la tesis. La demostración del caso infinito que Arquímedes requería es análoga, sólo que ahora el centro de gravedad de los segmentos infinitesimales $\alpha(x) d x$ de $A$ se define como $\frac{1}{A} \int_{a}^{b} x \alpha(x) d x$. De esta manera, si los segmentos balancean a $B$ colocada en $-h, \circ \int_{a}^{b} x a(x) d x=h B$, entonces

$$
\bar{x} A=\frac{1}{A} \int_{a}^{b} x \alpha(x) d x \cdot A=\int_{a}^{b} x \alpha(x) d x=h B,
$$

con lo que se prueba la aserción.

Es así como concluimos nuestro análisis de las tres grandes suposiciones extraordinarias en las que está basado El método de Arquímedes. Como vimos, todas ellas son esencialmente correctas, por lo que no debe extrañar que el método heurístico de Arquímedes lo condujera a resultados verdaderos. Lo que sí causa asombro y admiración, sin embargo, es 
que un matemático que vivió en el siglo ni a. c. haya podido ver su validez.

\section{Los resultados del método}

Utilizando su método, Arquímedes descubrió o se convenció de la verdad de, al menos, las quince proposiciones enlistadas en $E M$. Todas ellas afirman que el área o volumen de alguna(s) figura(s) curva(s) es proporcional al área o volumen de otra figura (no necesariamente rectilínea), con la excepción de las proposiciones $5,6,8$ y 9 , las que afirman que el centro de gravedad de una figura dada es un punto dentro de la figura. Será suficiente analizar la investigación de una de estas proposiciones para obtener una idea adecuada del método de Arquímedes. Consedere la siguiente.

Proposición 1 de EM. Sea $A B C$ un segmento de una parábola acotado por la línea recta $A C$ y la parábola $A B C$, y sea $D$ el punto medio de $A C$. Dibuje una línea recta $D B E$ paralela al eje de la parábola y junte $A B, B C$.

Entonces el segmento $A B C$ será $4 / 3$ del triángulo $A B C$.

Desde $A$ dibuje $A K F$ paralela a $D E$, y deje que la tangente a la parábola en $C$ intersecte a $D B E$ en $E$ y a $A K F$ en $F$. Produzca $C B$ intersectando $A F$ en $K$, y nuevamente produzca $C K$ hacia $H$, haciendo $K H$ igual a $C K$ (vea la figura 8).

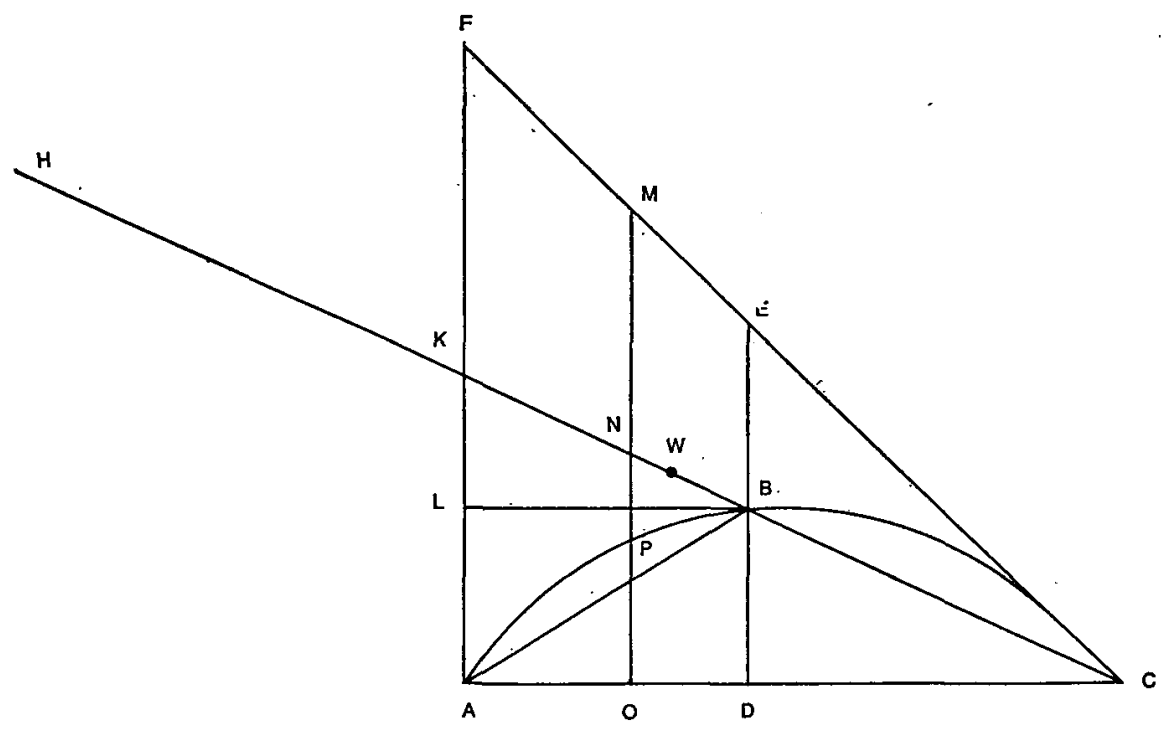

Figura 8. 
Lo que Arquímedes tenía que mostrar es que cada uno de los segmentos $O P$, paralelos a $F A$, ubicados con sus centros de gravedad en $H$, balancean el correspondiente segmento $O M$, con su centro de gravedad en $K C$, alrededor de $K$ considerado como el fulcro de una palanca, i. e., necesitaba mostrar que

$$
M O: O P=H K: K N
$$

donde $N$ es un punto variando sobre $K C$. Esto lo muestra Arquímedes como sigue: Dado que $C E$ es tangente a la parábola y $B D$ es la semiordenada (paralela al eje), el lema EM1 (ver el Apéndice) implica

$$
E B=B D .
$$

Así, dado que $F A, M O$ son paralelos a $E D$, se sigue (Euclides VI, 4) que

$$
F K=K A
$$

$$
M N=N O
$$

Ahora bien, dado que $A C$ es la base del segmento $A B C$ y el diámetro a través de $P$ intersecta $C A$ en $O$ y la tangente por $C$ en $M$, se sigue que

Asi,

$$
C O: O A=M P: P O \text {. }
$$

$$
\begin{gathered}
M P+P O: P O=C O+O A: O A \\
M O: O P=C A: A O .
\end{gathered}
$$

Considere ahora el triángulo $A C K$ y observe que $O N$ es paralela a $A K$; esto implica que

$$
\begin{aligned}
A O: O C & =K N: N C \\
C O: O A & =C N: N K \\
C O+O A: O A & =C N+N K: N K \\
C A: O A & =C K: N K \\
M O: O P & =C K: K N
\end{aligned}
$$

(Euclides VI, 2)

(invirtiendo)

(componiendo)

Como $H K=C K$,

$$
M O: O P=H K: K N
$$


que es la igualdad deseada. Puesto que $M O$ fue escogido arbitrariamente, Arquímedes ha mostrado realmente que "para todas las otras líneas rectas paralelas a $D E$ y que intersectan el arco de la parábola":

“(1) la porción interceptada entre $F C, A C$ con su punto medio sobre $K C$ y (2) una longitud igual a la interceptada entre la curva y $A C$ colocada con su centro de gravedad en $H$ estará en equilibrio alrededor de $K . "$

En este punto Arquímedes usa su suposición S2, pues concluye: "Por lo tanto,

(14) $K$ es el centro de gravedad del sistema completo consistente (1) de todas las líneas rectas como $M O$ interceptadas entre $F C, A C$ y ubicadas donde de hecho están en la figura y (2) de todas las líneas rectas ubicadas en $H$ iguales a las líneas rectas como $P O$ entre la curva y $A C . "$

En otras palabras, las líneas rectas como $M O$, ubicadas donde están en la figura, balancean alrededor de $K$ a las líneas rectas como $P O$, a las que podemos concebir aquí como una sola magnitud compuesta ubicada con su centro de gravedad en $H$. Por otro lado, "dado que

(15) el triángulo $C F A$ está compuesto por todas las líneas paralelas como $M O^{\prime \prime}(S 1)$

$\mathrm{y}$

(16) "el segmento [de parábola] está compuesto por todas las líneas rectas como $P O$ dentro de la curva" (SI),

"se sigue que

(17) el triángulo, ubicado donde está en la figura, está en equilibrio alrededor de $K$ con el segmento $C B A$ ubicado con su centro de gravedad en $H^{\prime \prime}$.

Al llegar este momento, Arquímedes aplica la suposición $S 3$ concibiendo el triángulo como si toda su magnitud estuviese concentrada en su centro de gravedad $W$, de manera que

$$
\triangle A C F \text { :segmento } A B C=H K: K W
$$

Ahora bien, el lector puede verificar que el lema EM4 implica que la línea $C K$ es a $K W$ lo que 3 es a 1; luego, puesto que $H K=C K$, 


$$
H K: K W=3: 1
$$

lo que, junto con (18), implica

$$
\triangle A C F: \text { segmento } A B C=3: 1
$$

Por otro lado, dado que los triángulos $K F C$ y $A K C$ están sobre bases iguales $A K$ y $K F$, y en las mismas paralelas, $A F$ y la paralela a $A F$ a través de $C$, se sigue que $\triangle K F C=\triangle A K C$ (Euclides I, 38). Por la misma razón, dado que los triángulos $A B D$ y $D B C$ se hallan sobre bases iguales $A D, D C$, también son iguales. Ahora, a través de un punto $L$ sobre $K A$, dibuje una paralela a $A D$ (Euclides I, 31) completando el paralelogramo $\angle B D A$; entonces el paralelogramo $L B D A$ es el doble del triángulo $A B D$, pues tienen la misma base $A D$ y están en las mismas paralelas $A K, B D$ (Euclides I, 41). Más aun, dado que $A F$ es paralela a $D E$ y $L B$ es paralela a $A C$, el ángulo $A K B$ es igual al ángulo $D B C$ y el ángulo $K B L$ es igual al ángulo $B C D$ (Euclides I, 29); pero $L B$, que es igual a $D C$, subtiende el ángulo $A K B$, de modo que el triángulo $L K B$ es igual al triángulo $B D C$ (Euclides $\mathrm{I}, 26$ ). Por ende, el triángulo $A K C$ es al triángulo $D B C$ lo que 4 es a 1 y

$$
\triangle A C F=4 \triangle A B C
$$

Por lo tanto,

$$
4 \triangle A B C \text { :segmento } A B C=3: 1
$$

$$
{ }_{\mathrm{3}} \triangle A B C=\text { segmento } A B C \text {. }
$$

El resultado (23) concluye la investigación de la Proposición 1 de EM. Aunque ahora sabemos que el procedimiento anterior en realidad demuestra la proposición, Arquímedes dice que

el hecho aquí enunciado en realidad no se prueba con el argumento usado; pero ese argumento ha dado una suerte de indicación de que la conclusión es verdadera. Viendo que el teorema no se demuestra, pero al mismo tiempo sospechando que la conclusión es verdadera, tendremos acceso a la demostración geométrica que yo mismo he descubierto y publicado. ${ }^{18}$

La proposición 1 de EM es formulada como Proposición 17 de Cuadratura de la parábola, seguida por la demostración geométrica aludida

18 Heath (1912), pp. S-17 y S-18. 
por Arquímedes en el pasaje anterior. ¿Qué tiene esta demostración que no tenga la de EM?

Dijksterhuis (1957) alega que la deficiencia matemática de los argumentos en EM es "exclusivamente una consecuencia del uso de indivisibles". ${ }^{19}$ Me parece que Dijksterhuis acierta al implicar que el uso de los indivisibles en los argumentos de EM los torna deficientes desde el punto de vista de la matemática griega: como vimos, aparte de que la suposición $S 1$ es en rigor falsa, la demostración de $S 2$ y $S 3$ era algo que se encontraba más allá de las posibilidades técnicas de los geómetras griegos, y claro que desde el punto de vista de ellos el uso de una suposición no justificada en un argumento hace al argumento defectuoso. Sin embargo, Knorr (1978) sugiere otra razón que también invalidaría el método de Arquímedes qua método de demostración (no de investigación). Ésta tiene que ver con uno de los cánones formales de demostración de la matemática griega, según el cual es permisible demostrar teoremas de una ciencia posterior utilizando principios de una anterior, pero no viceversa. ${ }^{20}$ Así, no es permisible utilizar los principios y resultados de la mecánica de los equilibrios y centros de gravedad para demostrar teoeremas geométricos, porque la primera ciencia es posterior a la geometría. Lo que determina esta prioridad entre las ciencias es la complejidad de las cosas estudiadas; por ejemplo, en el caso que nos ocupa, la geometría es anterior a la mecánica porque la primera atribuye a sus objetos sólo magnitud, mientras que la segunda les atribuye, además de magnitud, peso. ${ }^{21}$

\section{APÉNDICE}

Para comodidad del lector enuncio aquí cuatro de los lemas empleados por Arquímedes en El método.

(EM1) Si en una parábola $Q Q^{\prime}$ es una cuerda paralela a la tangente por $P$, y si se dibuja una línea recta a través de $P$ que es el eje o paralela al eje, y que intersecta a $Q Q^{\prime}$ en $V$ y a la tangente por $Q$ a la parábola en $T$, entonces $P V=P T$ (Figura 9).

(EM2) (Proposición I de Sobre conoides y esferoides). Si $A_{1}, B_{1}, C_{1}, \ldots$, $K_{1}$ y $A_{2}, B_{2}, C_{2}, \ldots, K_{2}$ son dos series de magnitudes tales que

19 P. 319.

20 Cfr. Aristóteles, Analiticos posteriores I, 6 y 7.

21 Suponiendo que la densidad de las figuras geométricas es igual a 1 , hemos seguido a Arquímedes al identificar su peso con su magnitud. 


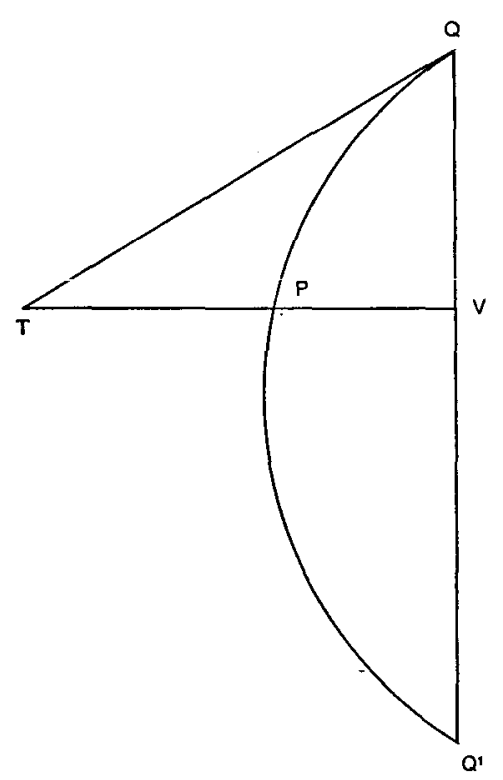

Figura 9.

$$
\begin{aligned}
& A_{1}: B_{1}=A_{2}: B_{2} \\
& B_{1}: C_{1}=B_{2}: C_{2}, \text { y así consecutivamente; }
\end{aligned}
$$

y si $A_{3}, B_{3}, C_{3}, \ldots, K_{3}$ y $A_{4}, B_{4}, C_{4}, \ldots, K_{4}$ son otras dos series tales que

$$
\begin{aligned}
& A_{1}: A_{3}=A_{2}: A_{4} \\
& B_{1}: B_{3}=B_{2}: B_{4}, \text { y así consecutivamente, }
\end{aligned}
$$

entonces $\left(A_{1}+B_{1}+C_{1}+\ldots+K_{1}\right):\left(A_{3}+B_{3}+C_{3}+\ldots+K_{3}\right)=\left(A_{2}+\right.$ $\left.B_{2}+C_{2}+\ldots+K_{2}\right):\left(A_{4}+B_{4}+C_{4}+\ldots+K_{4}\right)$.

(EM3) (Proposición 5 de Cuadratura de la parábola). Si $Q q$ es la base de cualquier segmento de parábola, $P$ el vértice del segmento, y $P V$ su diámetro, y si el diámetro de la parábola a través de cualquier otro punto $R$ intersecta $Q q$ en $O$ y a la tangente por $Q$ en $E$, entonces $Q O: O q=E R: R O$. (Figura 10).

(EM4) (Cfr. Sobre el equilibrio de los planos I, 14 y 15). El centro de gravedad de cualquier triángulo está en la intersección de las líneas dibujadas desde dos ángulos cualesquiera hacia los puntos medios de los respectivos lados opuestos y cualquiera de estas líneas es a su porción comprendida entre el lado y el centro de gravedad lo que 3 es a 1 . 


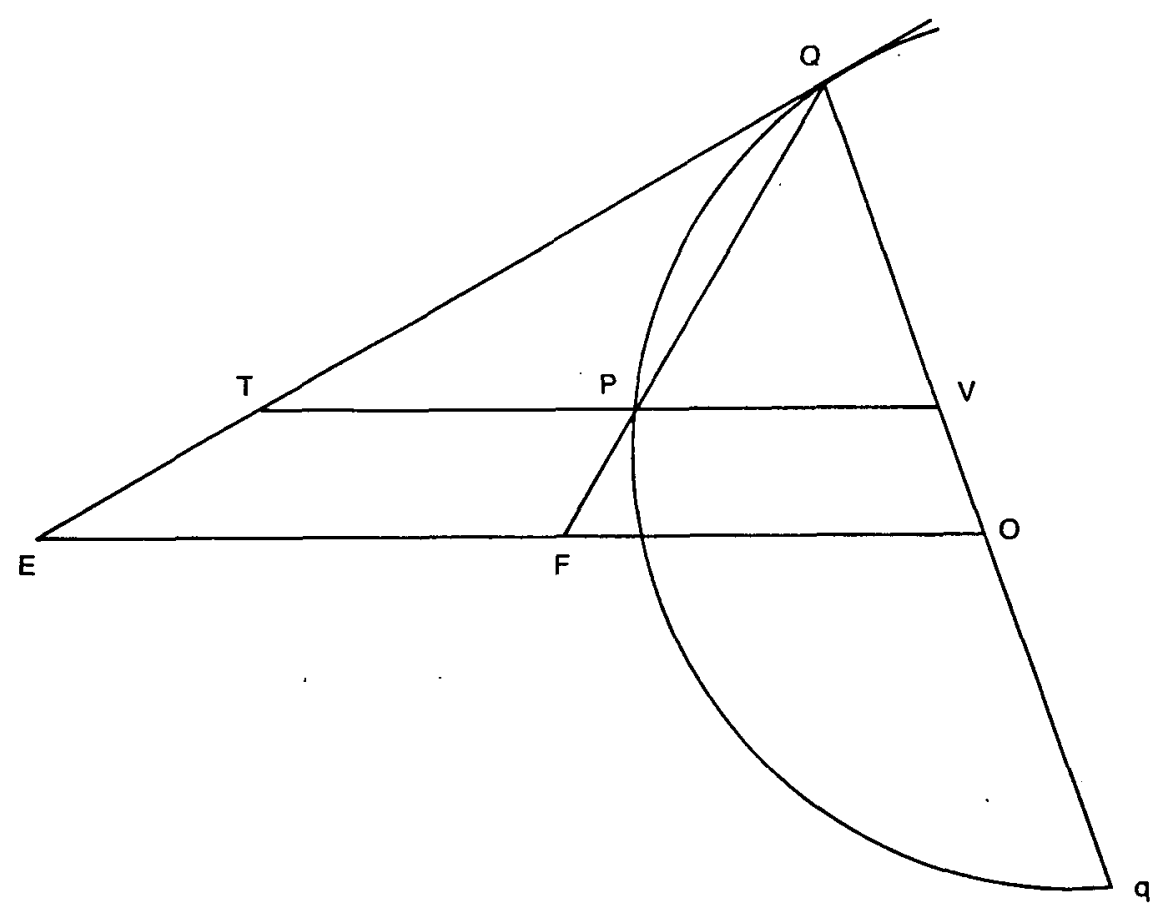

Figura 10.

\section{BIBLIOGRAFfA}

E. J. Dijksterhuis, Archimedes, New York, 1957.

T. L. Heath, The Works of Archimedes, New York, 1912.

- A History of Greek Mathematics, Oxford, 1921.

- Euclid. The Elements, New York, 1956.

J. L. Heiberg, "Eine neue Archimedeshandschrift", Hermes XLII, 1907.

J. M. Henle y E. M. Kleinberg, Infinitesimal Calculus, Cambridge, 1979.

Heronis Alexandrini Opera quae supersunt omnia, Leipzig 1900, 1903, 1912 y 1914.

W. R. Knorr, "Archimedes and the Elements: Proposal for a Revised Chronological Ordering of the Archimedian Corpus", Archive for History of Exact Sciences, v. 19, n. 3, 1978.

- "Archimedes' Lost Treatise on the Centers of Gravity of Solids", The Mathematical Intelligencer, v. 1, n. 2, 1978 a.

R. Mckeon, The Basic Works of Aristotle, New York, 1941.

W. Stein, Der Begriff des Schwerpunktes bei Archimedes, Berlin, 1931. 